\title{
Consumer Preferences for the Interface of E-Commerce Product Recommendation System
}

\author{
Yi-Cheng $\mathrm{Ku}^{1}$, Chih-Hung Peng ${ }^{2}$, and Ya-Chi Yang ${ }^{1}$ \\ ${ }^{1}$ Dept. of Computer Science and Information Management, Providence University, Taiwan \\ ycku@pu.edu.tw \\ ${ }^{2}$ Department of Information Systems, City University of Hong Kong, China \\ chpeng@cityu.edu.hk
}

\begin{abstract}
A recommendation system (RS) in a website is increasingly significant for consumer's decision making. A RS includes several important benefits, such as increasing user satisfaction and building user trust. Despite the growing literature that examined the usefulness of a specific attribute of a RS, less is known about which combination of attributes of a RS is preferable and how the combination influences consumer decision making. By using a conjoint analysis, we can further explore the impacts of combination attributes. In a lab experiment, we find that the importance ranking of attributes of a RS for the participants is quite different. Specifically, all the participants consider the attribute, "Explanation for Recommendation", is important. In addition, "Rating" is important for the specific participants. Furthermore, "Comment" seems to be less important to all the participants. Our results have important implications for the design of a RS.
\end{abstract}

Keywords: recommendation system, user interface preferences, adaptive interface, conjoint analysis.

\section{Introduction}

A recommendation system (RS) has played an increasingly important role in the success of e-commerce websites. A RS helps online customers facilitate their product searching and improve decision quality. In addition, through explicit or implicit learning, a RS can predict consumers' product preferences and provide customized recommendations. These benefits of a RS increase customers' satisfaction and trust toward the RS [1]. A RS is beneficial for not only consumers but also an e-commerce website. According to the report from Experian Marketing Services, the total revenue at Amazon.com in 2012 was $\$ 61.1$ billion, and around $30 \%$ of its total revenue came from product recommendations. Therefore, how to increase RS performance is a critical issue for practitioners and researchers.

Most previous studies have measured RS performance by either objective prediction accuracy (e.g. mean absolute error) or users' subjective perception (e.g. user satisfaction) [2]. However, these two measures pay less attention to the utility of interaction between users and a RS (i.e., user experience). Considering user experience is 
necessary because accuracy is not the only one criterion to evaluate an information system. Memorable experience will enhance users' continuous usage intention [3]. Hence, researchers have proposed a novel measure of RS performance by including the concept of user experience [3-5].

Our study focuses on designing a user interface of a RS. Although previous studies have proposed several guidelines for designing a user interface of a RS, most of the studies have focused on one recommendation mechanism at a time rather a combination of recommendation mechanisms (e.g., average ratings, associate products, expert review, and other related recommendations). However, when users are browsing a $\mathrm{RS}$, the interface of a RS is composed of multiple mechanisms instead of a single mechanism. Therefore, investigating a combination of recommendation mechanisms in an RS interface is reasonable and needed. The purposes of our research are twofold: First, we conduct a conjoint analysis to explore how users prefer different RS interfaces. Second, we explore the importance ranking of RS mechanisms for different user populations.

The rest of this study was organized as follows. First, we review related literature and Amazon.com to understand the principles of user interface design pertaining to RSs in section 2. Then, the research methods are described in section 3 . In section 4 , the results of conjoint analysis and research findings are discussed. We make a brief conclusion in final section.

\section{Literature Review}

Prior studies have proposed several principles to design a user interface. A user interface is defined as a platform which allows users to interact with a system. The first principle is to keep a user interface simple. Specifically, a user interface should only include necessary information, and information included should be concise. The second principle is related to an interface representation. Users spend less cognitive effort interacting with an interface when they use a visual representation interface than a text representation interface. To reduce user's cognition burden, interface designers can replace texts with simple icons on an interface. For instance, a thumb up emoticon means "recommended", while a thumb down one means "not recommended".

These principles have suggested that interface designers can design a better interface for users when they understand how users interact with an interface by minimum cognitive effort. Based on cognitive load theory, humans keep minimum load on working memory to seek optimum learning [6]. That is, optimum interface which provides easily recognized, sufficiently and necessarily detailed interface can reduce RS users' cognitive load [7]. Previous studies have explored how interface contents should be presented. For instance, Lee and Benbasat [8] have shown that the size and clarity of product pictures on the interface affect users' memory of products. Cai and $\mathrm{Xu}$ [9] have proposed that different arrangements have different impacts on users. They found that users tend to buy higher quality products when products are sorted by a quality criterion in a descending order than in an ascending order. 


\subsection{RS Interface Design}

A user interface of a RS is the key to affecting whether users are willing to use the system continuously. An adaptive RS interface can increase such willingness because users can find products interested efficiently and effectively. Previous studies have proposed several important mechanisms which are used in designing an interface of a RS. Wang and Benbasat [1] found that users have a higher level of initial trust in a RS with an explanation mechanism than a RS without it. If a RS offers explanation why a product was recommended, users' understanding and acceptance of the system will increase [10]. They will find that the process of recommendation is useful, and different degrees of transparency will provide them with different experiences. In addition, Jones and $\mathrm{Pu}$ [11] have suggested that a successful design of a RS should focus on a simple and clear interface that can reduce users' decision effort (e.g. the time spent on acquiring recommendation) and maximize the quality of recommendation (e.g. precision, satisfaction and novelty). In this study, we focus on how to design an adaptive interface of a RS by considering a set of mechanisms together.

We used Amazon.com to identify which general mechanisms are used often in a RS. Since Amazon.com is a global and large-scale e-retailer and many previous studies investigated the effects of RS by using Amazon.com [12], mechanisms provided by the RS of Amazon.com are typical and applicable. Based on recommendation page at Amazon.com, as shown in Fig. 1, our study identified six general attributes, including rating, explanation, associate products, the number of recommended products, customer review, and review comment.

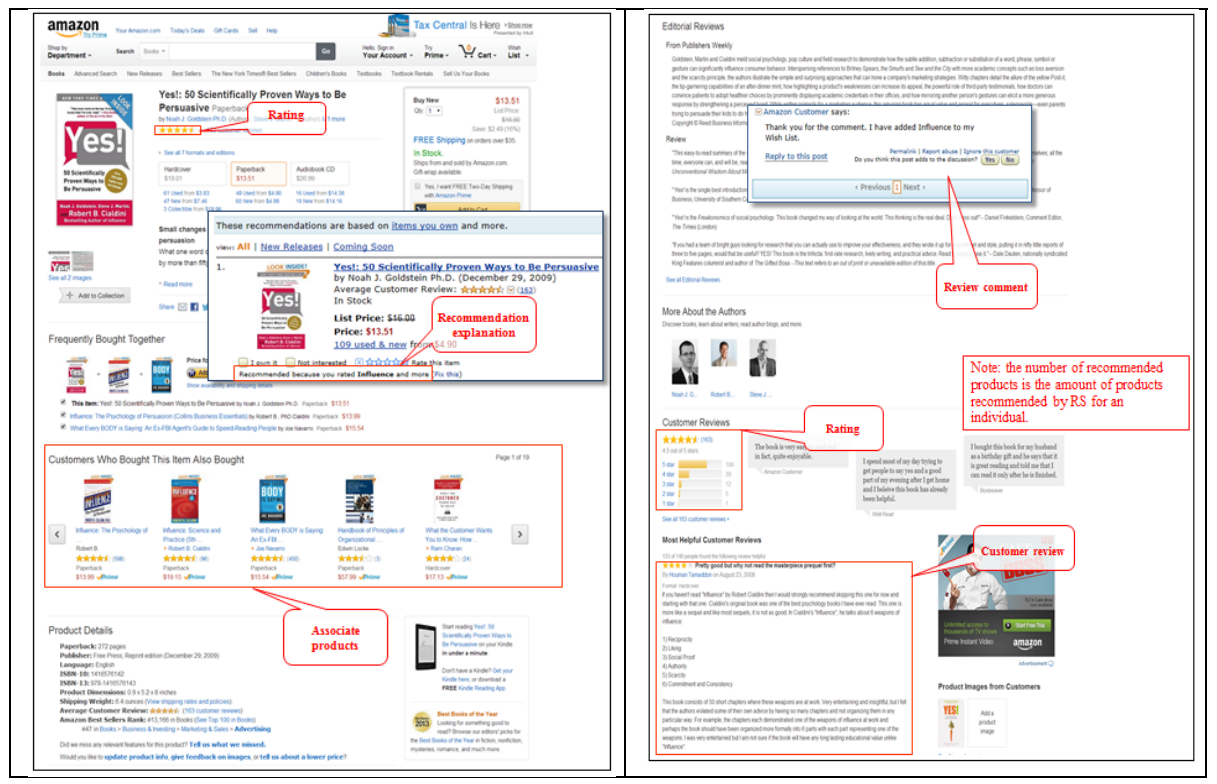

Fig. 1. Attributes in RS interface (An example of Amazon.com) 
When a user has limited knowledge of a field or in a field with a variety of goods, visual representation can effectively increase recommendation effectiveness and user's satisfaction. It can also attract users to use the comments more often without increasing their cognitive load [13]. Since the area of a web page is limited, it's important to adaptively present recommendation message. In particular, RS has been applied to mobile device which has smaller screen than monitor, it's necessary to understand RS users' priority in interface attributes and format. Since there are six attributes with different levels, this study adopted the conjoint analysis to evaluate users' preferences for the interface design of a RS.

\subsection{Conjoint Analysis}

When consumers are facing with a variety of products including goods and services, they would evaluate every attribute of products to make the final decision, and consumers will also establish a preference for a certain product in the process. Conjoint analysis is a multivariate method that uses the final decision and goes through the decomposition process to evaluate customers' preference structure. For example, conjoint analysis was used to explore shopping attribute combinations of website's characteristics preferred by consumers in order to offer reference basis for constructing websites [14]. Hence, conjoint analysis can be applied to understand how products with multiple attributes affect consumers' preferences. It contains all tangible and intangible functions of products, and it is able to pre-determine consumers' reaction and evaluation of potential product attribute combination.

Researchers often use a conjoint analysis to evaluate consumers' preferences of a certain product attribute combination. Subjects evaluate different product attribute combinations and their preference structure will then be decomposed, adding the partworth of each attribute under different levels to obtain an overall utilities. The product with the highest overall utilities is favored by consumers, and it has higher opportunity of being chosen. Besides obtaining the overall utilities, we can also calculate the relative importance of each attribute, which can explain each attribute's importance to the overall preferences. Hence, conjoint analysis was applied to design an adaptive IS interface. For example, Seneler et al. [15] adopted conjoint analysis to explore the interface feature prioritization for web services. Based on an experiment study, they found that interfaces that have high-speed, minimal memory load, adaptive behavior, low content density, and customization features are more preferable than those that do not. Therefore, this study used a conjoint analysis to explore users' preference for RS interface combination and prioritization of attributes.

\section{$3 \quad$ Research Method}

\subsection{Prototypes of RS Interface}

In this study, we selected six attributes of a RS and each attribute has different levels to investigate RS users' attitudes and preferences toward a RS interface. 
Combinations of the attributes with different levels allow us to have several prototypes of a RS interface. We briefly describe these attributes and their levels.

Rating. RS rating scales influence the level of cognitive effort users exert when using a RS. A delicate scale requires users to increase their cognitive effort to process information. In contrast, a coarse scale reduces their cognitive effort, but discourages them from making a precise evaluation. Therefore, we propose three types of a rating scale: 2-point, 5-point, and 10-point.

Explanation. Previous studies have suggested that, when a RS explains why a product is recommended, users are more satisfied with the recommend product and have a higher level of trust toward the system [1]. Therefore, we propose two levels of an explanation mechanism. That is, a RS is with an explanation and without it.

Associate Products. Associate products are the products that are relevant to a recommended product. For example, associate products of a mobile phone could be its screen protectors. Consumers may be interested in associate products when browsing recommended products. However, what kind of associate products should be recommended is not clear. Thus, we propose three kinds of associate products, including related goods, goods of the same type, and other items customers bought after viewing the item.

The Number of Recommended Products. The number of recommended products which were generated by a RS influences users' shopping decisions. If there are too many recommendations, it will cause users information overload. If there are too few recommendations, the products which users like may not be in the recommendation list. Hence we propose three levels of the number of recommended products: 14 items, 21 items, and 28 items.

Customer's Review. Customer review is one of the main information sources for consumers to understand the quality of goods [16]. We argue that information richness of a review affects how a user judges a product. For example, it's difficult to describe the effects of cosmetic goods by text. It would be useful to present "before" and "after" pictures for cosmetic goods experiences, so that consumers can easily evaluate the goods. As a result, customer's review may be revealed by multi-media content. Since users' preference on the format of customer review may vary, there are two levels of customer's review attribute, including "literary description" and "literary description with pictures".

Review Comment. A review comment is an evaluation of a specific review. Users can judge whether a review is helpful based on its feedback [17]. Review comment can be presented in a simple way (e.g., agreed or not) or with rich contents (e.g., criticisms with voting), and it can also describe a user's viewpoint on a certain review. Therefore, our study investigates what kind of comments is attractive to users and proposes two levels of comment, i.e., "literary description" and dichotomous "helpful or not". 


\subsection{Conjoint Analysis Design and Survey}

According to the observation of Amazon.com and previous relevant studies, this study proposes $216(3 \times 2 \times 3 \times 3 \times 2 \times 2)$ interface combinations of RS interface types to explore the relationships between RS interface attributes and users' preference for RS interface design. Following Green et al. [18], we conducted the mixed model of conjoint analysis. First, we establish the prototypes (i.e., stimuli in conjoint analysis) based on the combination of each level of each attribute. Our research adopts the orthogonal design in SPSS software package. We reduce the number of stimuli in the overall outline from 216 to 20,4 of which are reserved observations that are mainly used to check the effectiveness of the models. Even though we've reduced the number of stimuli to 20, it will still lead to subjects' information overload if we give them all 20 stimuli for evaluation at a time, and this will reduce the accuracy of the information we collect. In order to solve this problem, our research uses 20 stimuli as a unit. Each unit will be randomly divided into four groups with five stimuli in each group, where one of the stimuli becomes the reserved stimulus and the other four are experimental stimuli. A snapshot of an example of stimuli is shown in Appendix 1. Every subject only needs to evaluate one group; that is, each subject evaluates no more than five stimuli, giving them 1-9 points, where 1 point means that their preference for the specific stimulus is the lowest and 9 points the highest. Since the purpose of our research is to explore users' preferences for interface design of RS, we adopt narrative and graphical presentation to describe stimuli. Narrative is used to explain the function of each attribute, while graphical presentation allows subjects to truly feel the presentation of the interface. In particular, we adopted a full-profile to present stimuli, which allows subjects to do preference evaluation based on the stimulus with the overall profile of recommendation message (Appendix 1).

Then we adopted a mixed model combining self-explicated preference model and traditional conjoint analysis model to conduct conjoint analysis. We collect the relative weights of attributes and the ideal rating of every level under all the attributes, and then establish the questionnaires with limited overall profile and demographic variables. All subjects were asked to arrange the order of the six attributes, including rating, explanation, associate products, the number of recommended products, customer review, and review comment. The order of the attributes represents their importance. Then we listed all the levels of each attribute, and then ask subjects to arrange the order based on their preferences. The order of the levels represents their importance. We adopted cellphones as the recommended products in the stimuli. There are two main reasons: One is that almost people have one or more cellphones, and they use cellphones frequently and thus are familiar with them. The other reason is that cellphones are unisex goods. Hence, it would be easy to recruit voluntary subjects.

A pretest was conduct to validate the questionnaire. 14 graduate students and 14 college students were recruited from a medium university in Taiwan to evaluate the prototype and the questionnaire. The modification of the questionnaire was to increase the design type combination of the stimulus versions, and the purpose of this modification is to mark the features of each stimulus, which allows subjects to quickly grasp the difference and makes it easier for them to answer the questions. All 236 
volunteers were recruited from a university and a government department in central Taiwan to participate in our survey. All subjects were asked to evaluate selfexplicated preference and a randomly assigned group of stimuli. Finally, 193 questionnaires were effectively completed. The valid ratio of our questionnaire is $81.78 \%$. Most subjects' age falls between 21 to 30 years old (58.55 \%) and females take up $63.21 \%$ of sample. As for Internet using experience, only $8.29 \%$ of the sample had less than 5-year Internet usage. Furthermore, $94.82 \%$ of the sample had online shopping experience. In particular, most subjects $(79.79 \%)$ had the experience of browsing the e-stores with RSs.

\section{$4 \quad$ Data Analysis}

\subsection{Market Segment}

In order to understand similar consumers' preference for the interface factors of a RS more precisely, we adopted the componential segmentation model to segment subjects. The componential segmentation model uses the self-explicated preference data of subjects to conduct cluster analysis. The value of self-explicated preference was calculated by relative weights of attributes $\mathrm{x}$ the ideal rating of every level under all the attributes. And then, we use ANOVA and discriminant analysis to identify the validity of clustering and to distinguish the capacity of the discriminant function. First, we use Ward's method of hierarchical clustering approach to do cluster analysis and find that the ideal number of cluster is three. Then, we use the K-means method of non-hierarchical cluster approach to divide subjects into three clusters, and the number of subjects in cluster A, B, C is 52(26.94\%), 79(40.93\%), and 62(32.13\%), respectively. Finally, we apply ANOVA analysis to test whether there are significant differences among these three clusters. The analysis results show that, based on the linear relationships of the 15 levels, the value of Pillai's Trace is $1.321(\mathrm{~F}=24.728$, $\mathrm{p}<0.05)$ and the value of Wilks' Lambda is $0.101(\mathrm{~F}=27.213, \mathrm{p}<0.05)$. It means that the average values of the three clusters in each attribute level are not equal. As Table 1 shown, we also conducted Duncan's post-hoc analysis to compare the mean difference between the three clusters in each attribute level. If there is no comma between two cluster codes, the mean difference is non-significant.

Discriminant analysis is used to identify the validity of clustering and the identification ability of the discriminant function after the grouping of samples. We used the segmentation results of the three clusters as the dependent variables and the 15 selfexplicated values of subjects as discriminant variables to do discriminant analysis. In this way, we would be able to identify the validity of clustering and the identification ability of the discriminant function. Both hit ratio and Press $\mathrm{Q}$ were used to test whether the result of the predicted clustering has discriminant ability. The hit ratio is $97.93 \%$, and Press Q is $362.37>6.63$, which means that the discriminant function has high discriminant ability. Therefore, we can infer that the consumers from different clusters have significant difference in their preference for the interface design combination of a RS. 
Table 1. The analysis of the difference in average value of each cluster

\begin{tabular}{|c|c|c|c|c|c|c|c|}
\hline Attribute & Level & $\begin{array}{c}\text { Cluster 1 } \\
(\mathrm{n}=52)\end{array}$ & $\begin{array}{c}\text { Cluster 2 } \\
(\mathrm{n}=79)\end{array}$ & $\begin{array}{c}\text { Cluster } 3 \\
(\mathrm{n}=62)\end{array}$ & $\mathrm{F}$ & p-value & Duncan \\
\hline \multirow{3}{*}{ Rating $\left(\mathrm{x}_{1}\right)$} & 2-point & 0.0447 & 0.0513 & 0.0910 & 44.555 & .000 & $(12,3)$ \\
\hline & 5-point & 0.0603 & 0.0727 & 0.1155 & 47.830 & .000 & $(1,2,3)$ \\
\hline & 10-point & 0.0424 & 0.0463 & 0.0562 & 3.342 & .037 & $(12,23)$ \\
\hline \multirow{2}{*}{ Explanation $\left(\mathrm{x}_{2}\right)$} & No & 0.0635 & 0.0843 & 0.0678 & 9.664 & .000 & $(13,2)$ \\
\hline & Yes & 0.1160 & 0.1546 & 0.1157 & 15.822 & .000 & $(31,2)$ \\
\hline \multirow{3}{*}{$\begin{array}{l}\text { Associate } \\
\text { Products }\left(\mathrm{x}_{3}\right)\end{array}$} & Related goods & 0.0391 & 0.0661 & 0.0349 & 22.211 & .000 & $(31,2)$ \\
\hline & $\begin{array}{l}\text { Goods of the } \\
\text { same type }\end{array}$ & 0.0505 & 0.0906 & 0.0421 & 41.457 & .000 & $(31,2)$ \\
\hline & $\begin{array}{l}\text { Other items } \\
\text { customers } \\
\text { bought after } \\
\text { viewing the item }\end{array}$ & 0.0322 & 0.0528 & 0.0328 & 12.646 & .000 & $(13,2)$ \\
\hline \multirow{3}{*}{$\begin{array}{l}\text { The number of } \\
\text { recommended } \\
\text { products } \quad\left(\mathrm{x}_{4}\right)\end{array}$} & 14 items & 0.0304 & 0.0595 & 0.0410 & 14.843 & .000 & $(13,2)$ \\
\hline & 21 items & 0.0259 & 0.0571 & 0.0330 & 26.889 & .000 & $(13,2)$ \\
\hline & 28 items & 0.0179 & 0.0412 & 0.0197 & 18.215 & .000 & $(13,2)$ \\
\hline \multirow[b]{2}{*}{$\begin{array}{l}\text { Customer } \\
\text { review }\left(\mathrm{x}_{5}\right)\end{array}$} & $\begin{array}{l}\text { Literary } \\
\text { description }\end{array}$ & 0.0855 & 0.0491 & 0.0765 & 47.858 & .000 & $(2,3,1)$ \\
\hline & $\begin{array}{l}\text { Literary } \\
\text { description with } \\
\text { pictures }\end{array}$ & 0.1709 & 0.0867 & 0.1416 & 121.187 & .000 & $(2,3,1)$ \\
\hline \multirow{2}{*}{$\begin{array}{l}\text { Review } \\
\text { comment }\left(\mathrm{x}_{6}\right)\end{array}$} & $\begin{array}{l}\text { Literary } \\
\text { description of } \\
\text { comment } \\
\end{array}$ & 0.1264 & 0.0462 & 0.0714 & 87.743 & .000 & $(2,3,1)$ \\
\hline & $\begin{array}{l}\text { Helpful or Not } \\
\text { Helpful }\end{array}$ & 0.0943 & 0.0415 & 0.0607 & 35.953 & .000 & $(2,3,1)$ \\
\hline
\end{tabular}

\subsection{Results of Conjoint Analysis}

We estimate the part-worth utilities of each segment on the basis of the mixed model of the conjoint analysis and the market segmentation model. As formula 1 shown, ordinary Least Square Regression (OSL) was adopted to estimate the parameters in order to obtain the regression coefficient, i.e., the estimation of part-worth.

$$
\begin{aligned}
y_{i h}= & a+b \hat{y}_{i h}+B_{11} x_{i 11}+B_{12} x_{i 12}+B_{21} x_{i 21}+B_{31} x_{i 31}+B_{32} x_{i 32}+ \\
& B_{41} x_{i 41}+B_{42} x_{i 42}+B_{51} x_{i 51}+B_{61} x_{i 61}
\end{aligned}
$$

$y_{i h}$ : The $h^{\text {th }}$ subject's overall preference value for the $i^{\text {th }}$ stimulus

$\hat{y}_{i h}$ : The $h^{\text {th }}$ subject's self-explicated preference value for the $i^{\text {th }}$ stimulus

$B_{a l}$ : Subject's estimated part-worth of attribute $a$ with level $l$

$$
(a=1,2, \ldots, n, l=1,2, \ldots, m)
$$

$x_{i a l}$ : A dummy variable which presents attribute $a$ with level $l$ in the $i^{\text {th }}$ stimulus (For instance: $\mathrm{X}_{11}=1$ and $\mathrm{X}_{12}=0$ was defined as 2-point $(1,0)$, while $\mathrm{X}_{11}=0$ and $\mathrm{X}_{12}=1$ was defined as 5-point $\left.(0,1)\right)$

Each subject has to evaluate one of the four groups pertaining to the stimulus unit and there are five stimuli in each group, but one of them is reserved observation value that won't be included in the regression equation. Therefore, each subject has five evaluation data of the overall profile and each level was coded by dummy variable. 
As a result, there are 52 subjects in the cluster 1 with 208 observation values, 79 in cluster 2 with 316 observation values, and 62 in cluster 3 with 248 observation values. The regression results of cluster 1 to cluster 3 are shown in Table 2 .

Table 2. The linear regression models in each segment

\begin{tabular}{|c|c|c|c|c|c|c|c|c|c|c|}
\hline \multirow{2}{*}{ Model } & \multirow{2}{*}{$\begin{array}{l}\text { Standardized } \\
\text { coefficient of } \\
\hat{\boldsymbol{y}}_{\boldsymbol{i h}}\end{array}$} & \multicolumn{8}{|c|}{ Standardized Coefficient of Dummy variables $\mathbf{X}_{\mathbf{1 1}} \sim \mathbf{X}_{\mathbf{6 1}}$} \\
\cline { 3 - 12 } & $\mathbf{X}_{\mathbf{1 1}}$ & $\mathbf{X}_{\mathbf{1 2}}$ & $\mathbf{X}_{\mathbf{2 1}}$ & $\mathbf{X}_{\mathbf{3 1}}$ & $\mathbf{X}_{\mathbf{3 2}}$ & $\mathbf{X}_{\mathbf{4 1}}$ & $\mathbf{X}_{\mathbf{4 2}}$ & $\mathbf{X}_{\mathbf{5 1}}$ & $\mathbf{X}_{\mathbf{6 1}}$ \\
\hline Cluster 1 & 0.092 & $0.136^{\varphi}$ & 0.129 & $0.161 *$ & $-0.162 *$ & -0.106 & 0.015 & $0.156^{*}$ & $-0.203 *$ & -0.046 \\
\hline Cluster 2 & 0.14 & -0.033 & $0.126^{\varphi}$ & 0.105 & 0.08 & 0.017 & -0.04 & 0.057 & $-0.134 *$ & 0.071 \\
\hline Cluster 3 & 0.161 & 0.048 & $0.158^{\varphi}$ & 0.081 & -0.08 & -0.078 & -0.054 & 0.062 & 0.002 & -0.034 \\
\hline
\end{tabular}

$* p<0.05 ; \varphi p<0.1$

All three regression models are reasonable because there is no autocorrelation between error terms ( $D-W$ value is between 1.5 and 2.5 ). The $\mathrm{R}^{2}$ of regression model for cluster 1 is $0.177(\mathrm{~F}=4.235, p<0.001)$. The result of our analysis shows that the subjects in cluster 1 have preference for the shopping websites that offer explanations for recommendation, 21 items at a time for the number of recommended products, and the 2-point rating. However, they have less preference for the websites that offer the recommendation of related goods and the review with only literary description. The $\mathrm{R}^{2}$ of regression model for cluster 2 is $0.118(\mathrm{~F}=4.091, p<0.001)$. The subjects in cluster 2 have preference for the 5-point rating. However, they have less preference for the websites that offer the review with only literary description. The $\mathrm{R}^{2}$ of regression model for cluster 3 is $0.079(\mathrm{~F}=2.044, p<0.05)$. The subjects in cluster 3 have preference for the 5-point rating. These three regression models were used to predict the subjects' preferences of reserved stimuli in each segment respectively. The results show that the explanation powers are acceptable.

\subsection{The Analysis of Part-Worth Utilities}

Based on the results of the discussions in the previous sub-section and the part-worth utilities of each attribute's base level being 0 , we can obtain a complete set of partworth utilities, which can represent the common preference structure of all subjects in a specific segment. In this sub-section, we are going to use the part-worth utilities of each segment to further explore the attribute's weight of each segment and the preference combination. According to Wind et al. [19], we use the differences between the part-worth utilities of levels within an attribute to estimate the relative importance of the attributes. The formula is as follows,

$$
R I A_{k}=\frac{A_{k}}{\sum_{p=1}^{p} A_{k}} \times 100 \%
$$

$\mathrm{RIA}_{\mathrm{k}}:$ the relative weight of the attribute $\mathrm{k}$

$\mathrm{A}_{\mathrm{k}}$ : the highest part-worth minus lowest part-worth within attribute $\mathrm{k}$ 
We can infer from the formula above that the greater difference between levels within an attribute is, the higher the relative weight of the attribute becomes. Thus, we can find out which changes in attribute levels can cause greater consumer preference variation. The top three most preferred attributes of the RS interface in each cluster was summarized in Table 3.

Table 3. Preferred attributes with preferred level

\begin{tabular}{|c|l|l|}
\hline Cluster & \multicolumn{1}{|c|}{ Preferred attributes } & \multicolumn{1}{c|}{ Preferred level } \\
\hline \multirow{3}{*}{ Cluster 1 } & Review (22.42\%) & literary description with pictures \\
\cline { 2 - 3 } & The number of recommended goods (20.00\%) & 21 items \\
\cline { 2 - 3 } & Explanation (17.94\%) & Giving explanations \\
\hline \multirow{3}{*}{ Cluster 2 } & Rating (24.26\%) & 5-point \\
\cline { 2 - 3 } & Review (22.11\%) & literary description with pictures \\
\cline { 2 - 3 } & Explanation1 (7.39\%) & Giving explanations \\
\hline \multirow{3}{*}{ Cluster 3 } & Rating (39.17\%) & 5-point \\
\cline { 2 - 3 } & Associate products (19.97\%) & customers' final purchase item \\
\cline { 2 - 3 } & Explanation (17.39\%) & Giving explanations \\
\hline
\end{tabular}

\section{Discussions and Conclusions}

A RS has been used as a tool that helps consumers make shopping decisions in ecommerce, and it has three advantages: turning Internet surfers into buyers, increasing product cross-selling opportunities, and securing customers' loyalty to the website. Most of the previous studies focused on the accuracy of RS. However, there are few studies investigating the effects of adaptive RS interface on users' usage behaviors. The results of this study not only have important practical insights but also provide researchers theoretical implications. Our research adopts a conjoint analysis to explore the difference in the consumers' preference for the attribute combination of a RS interface. Three clusters segmented by customer's self-explicated value were grouped and the ordinary least square regression which estimates part-worth utility of each attribute level was also proposed for each segment. In addition, we also examine the importance ranking of RS's attributes in different segments when RS users are browsing recommended messages. As for the rating, its importance in cluster 2 and cluster 3 is relatively high, and it's ranked No.1 in both. However, for the consumers in cluster 1, it's not an important attribute, and it's ranked No.5. Most subjects prefer the 5-point scale. About the customer review, the consumers in cluster 1 and cluster 2 put much emphasis on it, and its importance rank is No.1 and No.2 relatively. However, consumers in cluster 3 don't consider it to be an important attribute, and it is ranked No.6. Most consumers prefer the format of review to be presented in literary description with pictures.

About explanation, its attribute importance percentages in each segment are close, and the importance rank is 3 in all the segments. Providing explanation will enhance consumers' utilities in all segments. This result is consistent with the findings of previous studies which indicated that a RS with transparency can also affect users' perceived usefulness during the recommendation process and increase their acceptance and understanding of RS. Regarding to associate products and the number of 
recommended products, the consumers' preferences for these two attributes are inconsistent among the three segments. The rank of associate products is No. 4 in cluster 1 and cluster 2, and No. 2 in cluster 3 . The rank of the number of recommended products is No. 2 in cluster 1, No. 6 in cluster 2, and No. 4 in cluster 3. In addition, most consumers prefer the interface to show 21 recommended items at a time. Our findings indicate that RS users' preferences for RS interface are different. Website designer can enhance RS users' experience by providing adaptive interface. As for the comment function, its importance percentage and rank are relatively low in all the segments, and it's ranked No.6 in cluster 1, No.5 in cluster 2 and cluster 3 . These results indicate that consumers may consult customer reviews when they are evaluating the recommended products, but they may skip the comments which criticize the customer reviews.

Acknowledgements. This work was partially supported by the National Science Council of the Republic of China under the grant NSC 101-2410-H-126-005-MY2 and a grant from the City University of Hong Kong [Project No. 7200328].

\section{References}

1. Wang, W., Benbasat, I.: Recommendation Agents for Electronic Commerce: Effects of Explanation Facilities on Trusting Beliefs. Journal of Management Information Systems 23, 217-246 (2007)

2. Cremonesi, P., Garzotto, F., Turrin, R.: Investigating the Persuasion Potential of Recommender Systems from a Quality Perspective: An Empirical Study. ACM Transactions on Interactive Intelligent Systems 2, 1-41 (2012)

3. Knijnenburg, B.P., Willemsen, M.C., Gantner, Z., Soncu, H., Newell, C.: Explaining the User Experience of Recommender Systems. User Modeling and User-Adapted Interaction 22, 441-504 (2012)

4. Zins, A.H., Bauernfeind, U.: Explaining Online Purchase Planning Experiences with Recommender Websites. In: Frew, A.J. (ed.) Information and Communication Technologies in Tourism 2005, pp. 137-148. Springer Vienna (2005)

5. McNee, S.M., Riedl, J., Konstan, J.A.: Making Recommendations Better: An Analytic Model for Human-Recommender Interaction. In: CHI 2006 Extended Abstracts on Human Factors in Computing Systems, Montreal, Canada, pp. 1103-1108 (2006)

6. Sweller, J.: Cognitive Load during Problem Solving: Effects on Learning. Cognitive Science 12, 257-285 (1988)

7. Ozen, C., Basoglu, N.: Impact of Man-Machine Interaction Factors on Enterprise Resource Planning (ERP) Software Design. In: Proceedings of Portland International Conference for Management of Engineering and Technology 2006, Istanbul, Turkey, pp. 2335-2341 (2006)

8. Lee, Y.E., Benbasat, I.: Interface Design for Mobile Commerce. Communications of the ACM 46, 48-52 (2003)

9. Cai, S., Xu, Y.: Designing Product Lists for E-Commerce: the Effects of Sorting on Consumer Decision Making. Intl. Journal of Human-Computer Interaction 24, 700-721 (2008)

10. Gretzel, U., Fesenmaier, D.R.: Persuasion in Recommender Systems. International Journal of Electronic Commerce 11, 81-100 (2006) 
11. Jones, N., Pu, P.: User Technology Adoption Issues in Recommender Systems. In: Proceedings of the NAEC 2007, Riva del Garda, Italy, pp. 379-394 (2007)

12. Pathak, B., Garfinkel, R., Gopal, R.D., Venkatesan, R., Yin, F.: Empirical Analysis of the Impact of Recommender Systems on Sales. Journal of Management Information Systems 27, 159-188 (2010)

13. Hu, P.J.-H., Ma, P.-C., Chau, P.Y.K.: Evaluation of User Interface Designs for Information Retrieval Systems: A Computer-Based Experiment. Decision Support Systems 27, 125-143 (1999)

14. Chen, Y.H., Hsu, I.C., Lin, C.C.: Website Attributes That Increase Consumer Purchase Intention: a Conjoint Analysis. Journal of Business Research 63, 1007-1014 (2010)

15. Seneler, C.O., Basoglu, N., Daim, T.: Interface Feature Prioritization for Web Services: Case of Online Flight Reservations. Computers in Human Behavior 25, 862-877 (2009)

16. Hu, N., Liu, L., Zhang, J.J.: Do Online Reviews Affect Product Sales? The Role of Reviewer Characteristics and Temporal Effects. Information Technology and Management 9, 201-214 (2008)

17. Ku, Y.C., Wei, C.P., Hsiao, H.W.: To Whom Should I Listen? Finding Reputable Reviewers in Opinion-Sharing Communities. Decision Support Systems 53, 534-542 (2012)

18. Green, P.E., Goldberg, S.M., Montemayor, M.: A Hybrid Utility Estimation Model for Conjoint Analysis. Journal of Marketing 45, 33-41 (1981)

19. Wind, Y., Grashof, J.F., Goldhar, J.D.: Market-Based Guidelines for Design of Industrial Products. Journal of Marketing 42, 27-37 (1978)

\section{Appendix 1: A Sample Screen of Stimuli}

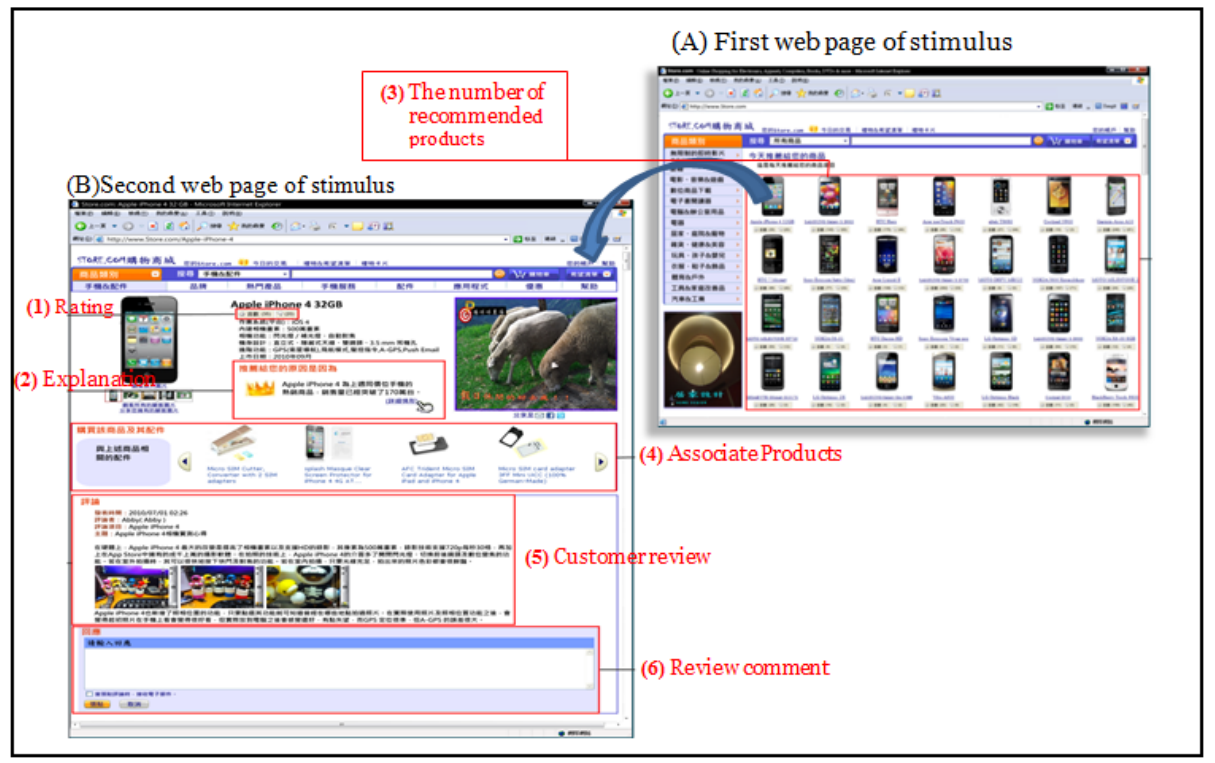

